\section{Immunochemical NMR}

\section{by a Correspondent in Molecular Biology}

NuClear magnetic resonance spectroscopy has not so far been much used in the study of proteins or indeed of nucleic acids. Its great potential in this field is only now being recognized more widely, and with improvements in instrumentation and the appearance during the past year or so of a number of strikingly successful applications, an increasing number of problems will be found to lend themselves particularly well to this approach.

The unique advantage of NMR for the study of polymers lies in its sensitivity to freedom of motion. Sharp signals are obtained only when the motion of the nuclei under observation (most commonly protons) is very rapid: this situation will obtain for small molecules in solution or for flexible polymers with rapid segmental motion-a denatured protein, for example. When the polymer becomes rigid, as in a globular protein or an $\alpha$-helical polypeptide, the signal broadens, sometimes to a degree at which it effectively disappears. The possibility exists, moreover, of identifying sharp signals with a particular class of protons, as, for example, those of particular amino-acid side chains in proteins. The possibilities for the study of conformational changes, with perhaps isolated observation of particular groups, will commend themselves for many purposes.

The NMR technique also offers a method for the observation of ligand-binding to macromolecules, because signals from the ligand nuclei will be broadened when the molecule is immobilized by binding to the macromolecule. A good example of the potential of the method in the protein field is given by Haugland et al. in the current issue of Biochemistry $(6,498 ; 1967)$. They have studied the interaction of an antibody with a hapten but with a more refined procedure which permits the use of minimal concentrations. Rather than proton resonance, they observe that of the chlorine $\left({ }^{35} \mathrm{Cl}\right)$ nucleus, which is present in the hapten. The hapten is a mercurial, 2,4-dinitro$4^{\prime}$-(chloromercuri)diphenylamine. The experiments are carried out in sodium chloride solution, and the existence of free chloride ions, which can exchange with the mercury-bound chlorine in the hapten, leads (as shown in an earlier article by Stengle and Baldeschwieler) to an amplified line-broadening effect. Because the hapten is added to the antibody, so a broadening of the ${ }^{35} \mathrm{Cl}$ resonance is observed, and it is possible to perform a titration by this means to give the stoichiometry of the association at antibody concentrations down to $6 \times 10^{-7} \mathrm{M}$. When dinitrophenyllysine is added to the complex, the linewidth again decreases, showing that the mercurial hapten is displaced.

The observed broadening of the signal when the mercurial hapten is bound defines a limiting rate of exchange of chlorine between hapten and solvent, and demonstrates that the hapten is fully exposed. The line-width will also reflect the rotational freedom of the chlorine in the hapten-that is to say, in effect the flexibility of the binding region. It is interesting to note that unbound hapten does not interfere with the measurements, and that weak intcractions can therefore be studied; the authors state that a 1 per cent level of binding can be detected.

\section{Nuclear Hyperfine Spectroscopy}

Professor J. F. Duncan writes: The majority of papers delivered at the International Conference on Nuclear Hyperfine Spectroscopy held at Victoria University of Wellington, New Zealand, from October 17-21, 1966, were concerned with Mössbauer spectroscopy, but there were also contributions on nuclear magnetic resonance and electron spin resonance. The conference was attended by visiting scientists from Australia, Brazil, the Congo, Germany, India, Israel, Japan, New Zealand, United Kingdom, U.S.A. and U.S.S.R., and consisted of four principal review papers and about forty short research papers.

The first of the review papers was given by Professor H. Frauenfelder, who described the use of Mössbauer spectroscopy in physics. New techniques, such as populating the excited state by coulombic excitation, or using scattering techniques which enable higher energy states to be more readily detected, were described. The second review paper by D. P. Craig outlined problems in calculating the interaction energies within a crystalline matrix. E. Fluck reviewed the current state of nuclear magnetic resonance spectroscopy of heavy nuclei, such as phosphorus-31, fluorine-19 and cobalt-59. The last review paper by V. J. Goldanskii was a most informative contribution on recent advances of Mössbauer spectroscopy in chemistry. It outlined the rapidly increasing applications in the field of structural determination, and discussed work carried out in Russia on investigating some biologically important compounds, including several components of RNA.

The research papers covered a wide range of topicstheoretical work, magnetic fields, chemical structure problems, solid state problems, instrumentation, general effects-and concluded with a most stimulating paper by $\mathrm{P}$. Hillman on Mössbauer studies on hearing.

The range of topics discussed in the research papers may be judged from the following brief summary. N. N. Greenwood assessed NMR techniques for measuring the stability of complexes; there are two methods for determining formation constants: the first can be applied where there is rapid exchange between free and complexed ligand, the second when there is also dissociation as well as exchange. Hyperfine interaction constants from NMR spectra of low spin ferric compounds was discussed by Golding and by Kurland. M. A. Collins discussed the measurement of nuclear hyperfine coupling constants in complex spectra. The calculation of $\mathrm{g}$ and hyperfine tensors for small molecules in double quantum transitions in ESR spectra was described by J. E. Wertz, and B. G. Wybourne discussed the use of pseudo-interactions in calculating spectroscopic properties and the effect of ionic contributions to S state rare-earth ions. A. J. F. Boyle spoke on relaxation and the Mössbauer effect in small magnetic particles, and gave a theoretical description of the effect of electron spin relaxation on the energy spectrum when there is an axis of quantization which remains fixed despite the time averaging introduced by the relaxation. J. J. Spijkerman and F. C. Ruegg have developed instrumentation for routine and high precision Mössbauer spectroscopy. R. E. Bailey and J. F. Duncan have shown that the three compounds $\mathrm{FeP}, \mathrm{Fe}_{2} \mathrm{P}$ and $\mathrm{Fe}_{3} \mathrm{P}$ fall into a regular array, and that the magnetic field at the iron atom is determined by 